07

\title{
Генерация СВЧ-колебаний \\ в сверхпроводниковой туннельной меза-структуре с прослойкой из ферромагнитного изолятора
}

\author{
() К.И. Константинян, Г.А. Овсянников, Ю.В. Кислинский, \\ А.М. Петржик, А.В. Шадрин
}

Институт радиотехники и электроники им. В.А. Котельникова РАН, Москва E-mail: karen@hitech.cplire.ru

Поступило в Редакцию 6 июня 2017 г.

\begin{abstract}
Экспериментально исследован спин-поляризованный ток в тонкопленочных туннельных меза-структурах, состоящих из эпитаксиальных пленок купратного сверхпроводника $\mathrm{YBa}_{2} \mathrm{Cu}_{3} \mathrm{O}_{7-\delta}$, манганита $\mathrm{LaMnO}_{3}$ и верхнего сверхпроводящего бислоя $\mathrm{Au}-\mathrm{Nb}$. Обнаружена собственная узкополосная генерация СВЧ-диапазона, частота которой перестраивается напряжением смещения и внешним магнитным полем.
\end{abstract}

DOI: 10.21883/PJTF.2017.20.45146.16904

В последнее время сверхпроводниковые структуры со спин-поляризованным током вызывают повышенный интерес ввиду перспектив их использования в устройствах спинтроники и новых элементов СВЧ-микроэлектроники. Проблема взаимодействия сверхпроводимости и магнетизма изучалась в гибридных структурах, содержащих сверхпроводники и ферромагнитные материалы, туннельных переходах из сверхпроводников с прослойкой из ферромагнитного изолятора (см., например, [1,2]). Туннельный ток, протекающий через ферромагнитную прослойку между двумя немагнитными электродами, оказывается спин-поляризованным вследствие расщепления энергетической зоны на состояния со спином вверх и спином вниз [3,4]. Возбуждение постоянным инжектированным спин-поляризованным током прецессии намагниченности ферромагнетика теоретически рассмотрено в работах [5-7]. Экспериментально возбуждение прецессии намагниченности при протекании достаточно высокой плотности тока (выше 
$\left.10^{6} \mathrm{~A} / \mathrm{cm}^{2}\right)$ наблюдалось в многослойных структурах из ферромагнетиков и металлов [8-10] и туннельной структуре из-за спинового эффекта Холла [11]. В то же время динамика спин-поляризованного тока в сверхпроводниковых структурах с ферромагнитной прослойкой остается пока малоизученной. Задачей настоящей работы является экспериментальное исследование сверхпроводниковой меза-структуры с ферромагнитной туннельной прослойкой, обладающей свойствами спинового фильтра.

Исследовались гибридные меза-структуры из эпитаксиальных пленок сверхпроводящего купрата $\mathrm{YBa}_{2} \mathrm{Cu}_{3} \mathrm{O}_{7-\delta}(\mathrm{YBCO})$ и манганита $\mathrm{LaMnO}_{3}$ (LMO) - материалов, имеющих хорошую химическую и кристаллическую совместимость [12]. Другим сверхпроводником был бислой, состоящий из $\mathrm{Nb}$ и тонкой пленки $\mathrm{Au}$, обеспечивающей контакт с манганитом. Сверхпроводящая пленка YВСО изготавливалась методом лазерной абляции при температуре $700-800^{\circ} \mathrm{C}$ на подложке (110) $\mathrm{NdGaO}_{3}$. Тонкая пленка из манганита LMO толщиной $d_{M}=5-20 \mathrm{~nm}$ эпитаксиально выращивалась в той же вакуумной камере (in situ) при высокой температуре и после охлаждения до комнатной температуры покрывалась слоем Аu толщиной 20-30 nm. Критическая температура пленки ҮВСО составляла $T_{C}=88-89 \mathrm{~K}$. Последующий слой $\mathrm{Nb}$ наносился методом магнетронного распыления. Пленка $\mathrm{Au}$ использовалась для уменьшения диффузии кислорода из манганита в $\mathrm{Nb}$. Эффект близости между пленками $\mathrm{Nb}$ и $\mathrm{Au}$ обеспечивал сверхпроводящую критическую температуру бислоя $T_{C^{\prime}}=8.5-9 \mathrm{~K}$, близкую к критической температуре пленки $\mathrm{Nb}(9.2 \mathrm{~K})$. Меза-структуры с размерами в плане от $10 \times 10$ до $50 \times 50 \mu \mathrm{m}$ с прослойкой из LMO (рис. 1) формировались с помощью фотолитографии, а также плазмохимического и ионно-лучевого травления. Согласно $[13,14]$, манганит LMO стехиометрического состава при низких температурах обладает свойствами изолятора и антиферромагнетика, однако небольшое изменение содержания кислорода приводит к возникновению ферромагнетизма с температурой Кюри $140-150 \mathrm{~K}$. О ферромагнетизме в пленках LMO сообщалось в работе [15], что было подтверждено измерениями ферромагнитного резонанса [16].

Исследование СВЧ-свойств меза-структур проводилось на установке, схематично показанной на рис. 1. Использовался холодный усилитель на транзисторах с высокой подвижностью носителей (НЕМТ), работающий в интервале частот $1-2 \mathrm{GHz}$. Усилитель имел собствен-

Письма в ЖТФ, 2017, том 43, вып. 20 


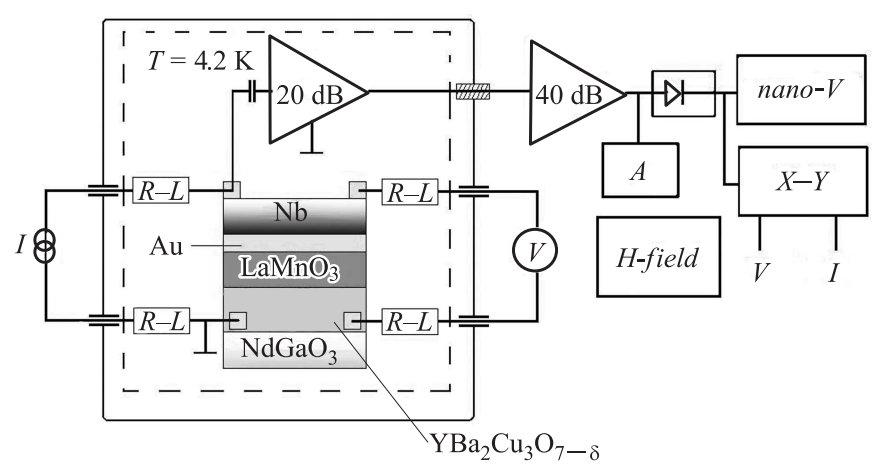

Рис. 1. Схема измерений вольт-амперных характеристик и СВЧ-генерации меза-структуры $\mathrm{Nb}-\mathrm{Au} / \mathrm{LaMnO}_{3} / \mathrm{YBa}_{2} \mathrm{Cu}_{3} \mathrm{O}_{7-\delta}$ на подложке $\mathrm{NdGaO}_{3}$. Мезаструктура (сечение показано в середине рисунка) и холодный усилитель размещались внутри соленоида задания магнитного поля (штриховой контур) и экрана (сплошной контур) при $T=4.2 \mathrm{~K}$. Напряжение смещения $I-V$ на меза-структуру, а также питание холодного усилителя задавались через $R-L$-фильтры и проходные конденсаторы. Усиленный СВЧ-сигнал от мезаструктуры подавался либо на анализатор спектра $(A)$, либо на квадратичный детектор. Уровень выходного сигнала контролировался нановольтметром (nano- $V$ ) и регистрировался графопостроителем $(X-Y)$.

ную шумовую температуру $T_{N 1}=8 \pm 2 \mathrm{~K}$ и коэффициент усиления $K_{1}=20 \mathrm{~dB}$ при охлаждении до температуры $T=4.2 \mathrm{~K}$. Балансная схема входного каскада обеспечивала устойчивую работу усилителя в широком диапазоне сопротивлений нагрузки $10-100 \Omega$ и понижала температуру фонового излучения, попадающего на меза-структуру по коаксиальному кабелю. Шумовая температура „комнатного“ усилителя была $T_{N 2}=130 \mathrm{~K}$, коэффициент усиления $K_{2}=40 \mathrm{~dB}$. Напряжение выходного сигнала, пропорциональное мощности $P$, снималось с выхода квадратичного детектора. Одновременно анализатором спектра контролировалась амплитудно-частотная характеристика на выходе „комнатного“ усилителя. Магнитное поле задавалось перпендикулярно плоскости подложки и, согласно расчетам [17], его воздействие для туннельного перехода квадратной формы было аналогичным воздействию параллельно заданного поля. 


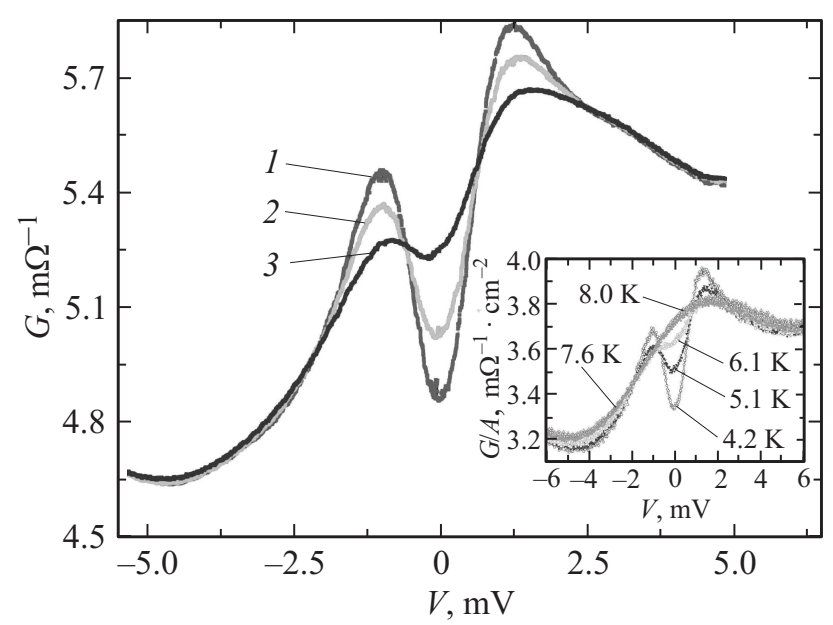

Рис. 2. Семейство зависимостей дифференциальной проводимости $G$ от напряжения меза-структуры с $d_{M}=5.6 \mathrm{~nm}$ и площадью $A=140 \mu \mathrm{m}^{2}$ при $T=4.2 \mathrm{~K}$ и значениях внешнего магнитного поля $H$, направленного параллельно плоскости подложки, равных 0 (1), 263 (2) и $526 \mathrm{Oе}(3)$. На вставке показана зависимость характерной проводимости $G / A$ от напряжения $V$ в диапазоне температур $4.2-8 \mathrm{~K}$ для $H=0$.

Измерение дифференциального сопротивления $R_{d}=d V / d I$ проводилось с помощью синхронного детектора и встроенного генератора модулирующего сигнала, задаваемого через цепь подачи постоянного тока, при этом магнитное поле задавалось параллельно плоскости подложки.

На рис. 2 представлены зависимости дифференциальной проводимости $G=1 / R_{d}$ при воздействии магнитного поля. Видна асимметрия $G(V)$ для положительных и отрицательных $V$, которая может быть вызвана формой туннельного барьера и разницей значений работ выхода YBCO и $\mathrm{Au}$. Наблюдается изменение $G(V)$ под влиянием магнитного поля в интервале напряжений до щели ниобия, которое трудно связать с зеемановским расщеплением плотности состояний электродов [13], поскольку напряженность магнитного поля $H$ существенно меньше поля зеемановского расщепления. После перехода в ферромагнитное состояние прослойки LMO при $T<140 \mathrm{~K}$ в меза-структуре возникает

Письма в ЖТФ, 2017, том 43, вып. 20 
спин-поляризованный ток [3]. Из рис. 2 видно, что магнитное поле влияет на компоненту туннельной проводимости, обусловленную спиновой поляризацией [14]. При увеличении напряжения постоянного смещения свыше $3 \mathrm{mV}$ (выше щели ниобия) проводимость меза-структуры уже не зависела от величины магнитного поля [16], а магнитосопротивление составляло величину, типичную для структур с манганитами [12]. Отметим, что магнитосопротивление обнаруживалось только при температурах ниже критической температуры ниобия.

На рис. 3, $a$ приведено семейство зависимостей мощности излучения от напряжения $P(V)$, полученных при нескольких значениях внешнего магнитного поля при $T=4.2 \mathrm{~K}$. При повышении температуры выше критической $T_{C^{\prime}}$ бислоя $\mathrm{Nb}-\mathrm{Au} \mathrm{CBЧ-генерация} \mathrm{исчезала.}$

Плавный рост $P$ с увеличением $V$, скорее всего, вызван дробовым шумом меза-структуры. Наблюдаются симметрично расположенные относительно $V=0$ выбросы $P(V)$, заметно превышающие уровень шума. На СВЧ-анализаторе спектра возникновение выбросов сигнала $P$ проявлялось в виде острого пика генерации с мощностью порядка долей $\mathrm{pW}$ и шириной $\sim 50 \mathrm{MHz}$. Центральная частота пика перестраивалась во всей полосе обзора $f=1-2 \mathrm{GHz}$ и росла с увеличением тока смещения $I$ (или напряжения $V$, как на рис. 3,a). Заметим, что в структурах $[8,9]$, напротив, частота генерации уменьшалась $\mathrm{c}$ увеличением тока $I$. Зависимость высоты мощности генерации $P$ от магнитного поля $H$ (при фиксированном $V$ ) имела осцилляционную форму со средним значением периода осцилляции порядка $0.5 \mathrm{Oe}$. Из рис. $3, a$ также видно, что интервал напряжений смещения $\Delta V$, при которых наблюдалась СВЧ-генерация, составлял в среднем $\Delta V \sim 8 \mathrm{mV}$. Оценка коэффициента перестройки частоты с изменением тока $\Delta f / \Delta I$ дает величину $7.5 \cdot 10^{12} \mathrm{~Hz} / \mathrm{A}$, где $\Delta I=\Delta V / R_{d}$, а величина $R_{d}$ составляет $60 \Omega$ и слабо зависит от $V$. Заметим, что отношение частоты генерации $f$ и соответствующего напряжения смещения $V$ на три порядка отличается от отношения $h f / 2 e V \cong 483.6 \mathrm{GHz} / \mathrm{mV}(h-$ постоянная Планка, $e-$ заряд электрона) для джозефсоновской генерации, которая наблюдается в контактах двух сверхпроводников. С учетом отсутствия сверхпроводящего тока и наблюдаемой в эксперименте связи частоты генерации и напряжения смещения на структуре объяснить такую СВЧ-генерацию нестационарным эффектом Джозефсона нельзя. 

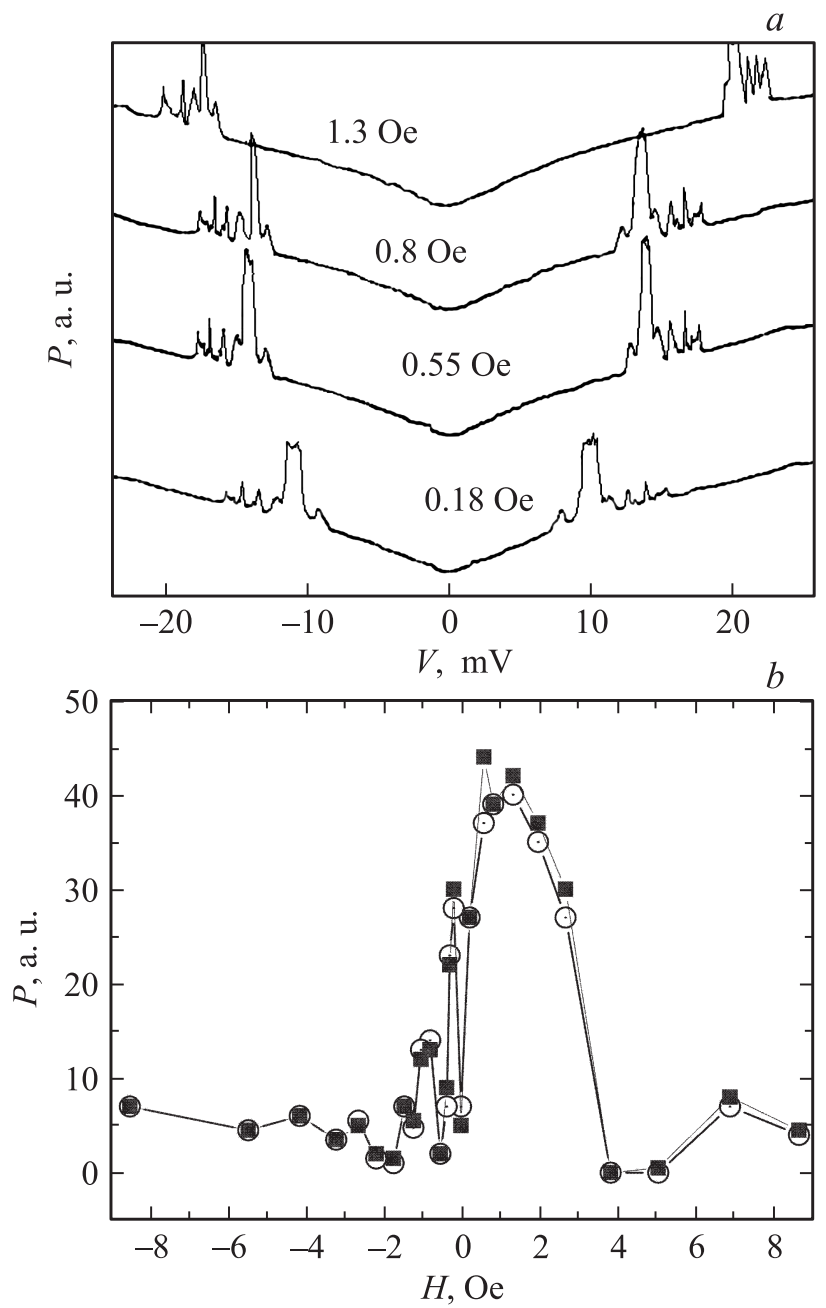

Pис. 3. $a-$ семейство зависимостей сигнала $P$ от напряжения смещения $V$ (сдвинуты по оси ординат), полученных с выхода квадратичного детектора при магнитных полях в диапазоне 0.18-1.3 Ое. Меза-структура имела размер $A=1000 \mu \mathrm{m}^{2}$ и толщину прослойки LMO $d_{M}=5.6 \mathrm{~nm}$, сопротивление $R=60 \Omega . b-$ зависимость максимума СВЧ-генерации $P$ от магнитного поля. Кружками обозначены пики, полученные при отрицательном смещении по $V$, квадратами - при положительном.

Письма в ЖТФ, 2017, том 43, вып. 20 
На рис. 3, $b$ показана магнитно-полевая зависимость максимальных значений амплитуд генерации $P$. Несмотря на то что зависимость амплитуды генерации от магнитного поля изрезана и имеет нерегулярную форму, значения максимумов, соответствующих обеим полярностям напряжения смещения, хорошо совпадают (рис. $3, b$ ).

Возникновение СВЧ-генерации можно было бы объяснить механизмом когерентного движения магнитных вихрей. Однако регулярное движение вихрей в сверхпроводящих пленках невоспроизводимо из-за влияния центров пиннинга, а кроме того, нарушается внешним СВЧ-воздействием [18]. Дополнительное воздействие на меза-структуру монохроматическим сигналом мощностью до $0.5 \mathrm{~mW}$ на частоте $f=70 \mathrm{GHz}$ незначительно (примерно на 20\%) подавляло амплитуду генерации, не изменяя ее форму. Более того, повторно прописанные зависимости $P(V)$ при фиксированном $H$ как с внешним СВЧ-сигналом $70 \mathrm{GHz}$, так и без него полностью совпадали, повторяя изрезанность $P(V)$.

Теоретически было показано [19], что управляемое вращение спиновых завихрений (скирмионов [20]) под действием постоянного спин-поляризованного тока может возбудить устойчивую узкополосную СВЧ-генерацию, и, что важно, без необходимого условия превышения порогового значения электрического тока. Согласно модели [19], в эксперименте наблюдается рост частоты генерации с увеличением тока через меза-структуру. Необходимое для образования спиновых завихрений сильное спин-орбитальное взаимодействие [20], относительно слабое в автономном манганите LMO [21], может быть усилено за счет ян-теллеровского искажения в меза-структуре из-за влияния нижней пленки YВСО [22], а также наличием прослойки из золота, спин-орбитальное взаимодействие в котором анализировалось в работе [23].

Таким образом, при изменении внешнего слабого магнитного поля $|H|<10$ Ое и тока смещения через меза-структуру $\mathrm{YBCO} / \mathrm{LMO} /$ $\mathrm{Au}-\mathrm{Nb}$, обладающую свойствами спинового фильтра, обнаружена СВЧ-генерация с шириной линии порядка $50 \mathrm{MHz}$. При этом частота генерации перестраивается током смещения с соотношением порядка $10^{13} \mathrm{~Hz} / \mathrm{A}$, а ее амплитуда управляется слабым магнитным полем $H \sim 0.5$ Oe.

Работа поддержана РАН, РФФИ (проект № 16-29-14022) и грантом Научной школы НШ-8168.2016.2. Авторы благодарны В.В. Демидову и И.В. Борисенко за обсуждение результатов.

Письма в ЖТФ, 2017, том 43, вып. 20 


\section{Список литературы}

[1] Bergeret F.S., Volkov A.F., Efetov K.B. // Rev. Mod. Phys. 2005. V. 77. P. 1321.

[2] Kawabata S., Tanaka Y., Golubov A.A. et al. // J. Magn. Magn. Mater. 2012. V. 324. P. 3467.

[3] Meservey R., Tedrow P.M. // Phys. Rep. 1994. V. 238. P. 173.

[4] Santos T.S. et al. // Phys. Rev. Lett. 2008. V. 101. P. 147201.

[5] Zhao E., Löfwander T., Sauls J.A. // Phys. Rev. B. 2004. V. 70. P. 134510.

[6] Slonczewski J.C.// J. Magn. Magn. Mater. 1996. V. 159. P. L1.

[7] Manchon A., Zhang S. // Phys. Rev. B. 2009. V. 79. P. 094422.

[8] Kiselev S.I., Sankey J.C., Krivorotov I.N. et al. // Nature (London). 2003. V. 425. P. 380.

[9] Rippard W.H., Pufall M.R. et al. // Phys. Rev. Lett. 2004. V. 92. P. 027201.

[10] Pribiag V.S., Krivorotov I.N., Fuchs G.D. et al. // Nature Phys. 2007. V. 3. P. 498.

[11] Liu Luqiao, Pai Chi-Feng et al. // Phys. Rev. Lett. 2012. V. 109. P. 186602.

[12] Петржик А.М., Овсянников Г.А., Шадрин А.В. и др. // ЖЭТФ. 2011. Т. 139. C. 1190.

[13] Moodera J.S., Santos T.S., Nagahama T. // J. Phys.: Condens. Matter. 2007. V. 19. P. 165202.

[14] Hao X., Moodera J.S., Meservey R. // Phys. Rev. B. 1990. V. 42. P. 8235.

[15] Renshaw X., Wang C.J., Li W., Lü M. et al. // Science 2015. V. 349. P. 717.

[16] Овсянников Г.А., Кислинский Ю.В. и др. // ЖЭТФ. 2017. Т. 151. С. 736.

[17] Monaco R., Aaroe M., Mygind J., Koshelets V.P. // Phys. Rev. B. 2009. V. 79. P. 144521.

[18] Константинян К.И., Овсянников Г.А. и др. // ЖЭТФ. 1991. Т. 72. С. 675.

[19] Garcia-Sanchez F., Sampaio J. et al. // New J. Phys. 2016. V. 18. P. 075011.

[20] Fert A., Cros V., Sampaio J. // Nature Nanotechnol. 2013. V. 8. P. 152.

[21] Snamina M., Oleś A.M. // Phys. Rev. B. 2016. V. 94. P. 214426.

[22] Борисенко И.В., Карпов М.А., Овсянников Г.А. // Письма в ЖТФ. 2013. T. 39. B. 23. C. 1.

[23] Dal Corso A., Conte A.M. // Phys. Rev. B. 2005. V. 71. P. 115106. 